\title{
ERRORS OF DIAGNOSIS REVEALED AT MENISCECTOMY
}

\author{
G. Murdoch, Dundee, Scotland \\ From the Royal Infirmary, Dundee
}

In the years $1940-55$ inclusive 2,668 semilunar cartilages were removed by surgeons working as a team and with a similar outlook on the internal derangements of the knee. The detailed records of the cartilages removed show that a relatively small proportionnamely 4.5 per cent-had no detectable lesion. This proportion nevertheless represents 119 cases. The purpose of this paper is to record the results of an investigation of the causes of error, in the belief that the incidence can be reduced.

The term " internal derangement of the knee joint" was first used by William Hey of Leeds in 1784 (Hey 1803), although Hippocrates was familiar with at least one of the conditions concerned. Nothing is known of their surgical treatment until the first record of the removal of a loose body by Paré (1558). It was not until 1877 that a torn semilunar cartilage was removed by Annandale of Edinburgh (Annandale 1885). In contrast, meniscectomy is now perhaps second only to operations for fractures of the neck of the femur on our operation lists.

The same difficulties in diagnosis that troubled the early workers beset us to-day, and normal cartilages continue to be excised in error. Martin (1913) reported an error of 4.5 per cent, Dunn (1934) of 13.66 per cent, Bristow (1935) of 7.2 per cent, and Smillie (1950) of 6.33 per cent. The cases in Smillie's report are included in the present series.

The great majority of cartilages removed show obvious tears, but some have to be closely examined before posterior horn erosions, horizontal cleavage or incomplete splits are revealed. There will however, be no real doubt in the surgeon's mind when he excises a normal cartilage in error. It is also true that experienced surgeons will continue to remove normal structures if damaged ones are not to be missed. This is inevitable in a condition where the reliability of the history is of paramount importance, and where so many other internal derangements exist to confuse.

Figure 1 shows the incidence of error year by year from 1940 to the present day. The increase in errors during the years 1941 to 1946 is explained in great part by the number of unreliable witnesses. With the end of the war many fewer cartilages were removed. At the same time the percentage error was halved. This improvement has been effected by a reduction in the number of malingerers, a careful approach to the problem of localisation, and the increased possibilities for delay in diagnosis. The final organisation of a fully integrated orthopaedic service has in turn brought about a further increase in the total number of cartilage operations performed yearly, but the error has been maintained at the low level of 3 per cent for nine years.

Investigation of the 119 normal cartilages removed shows many causes of error (Table I).

It is clearly of value to examine these errors in detail so that future mistakes may, if possible, be avoided.

Unreliable witnesses (thirty-two cases)-In this group thirty-two semilunar cartilages were removed in error from twenty-six patients. Of these patients twenty-two were considered to be malingerers (servicemen) or patients interested in compensation for alleged injury. The diagnosis of a torn cartilage depends so much on an accurate history that the surgeon may be misled by a carefully briefed patient, and more so in times of urgency such as a major war. Under normal conditions, however, the surgeon has the opportunity to delay a definite diagnosis, and to refer the patient to a rehabilitation unit to "prove" the knee. Of these twenty-two presumptive malingerers, six had both cartilages excised from the same knee 
at one operation. In all these cases doubt regarding the localisation of the lesion existed. Of the remaining sixteen patients six admitted that they had subjected themselves to operation in the knowledge that the joint was normal. Two patients, either before or after the operation in question, parted with all their four cartilages. One patient had had two removed previously and another, whose knee was explored for a retained posterior horn, had a complete intact cartilage removed. Four patients had had one cartilage excised previously, and of these three were known to be normal. All patients of this group gave typical histories of injury, locking, giving way and pain. In all, as might be expected, the anterior cruciate liagment was found to be normal at operation. Nineteen patients gave a history of clicking, but only in six cases was a click elicited by the examining surgeon.

The remaining four patients were all recorded before operation as unreliable witnesses and unable to relax during examination; one patient, in addition, presented a language difficulty.

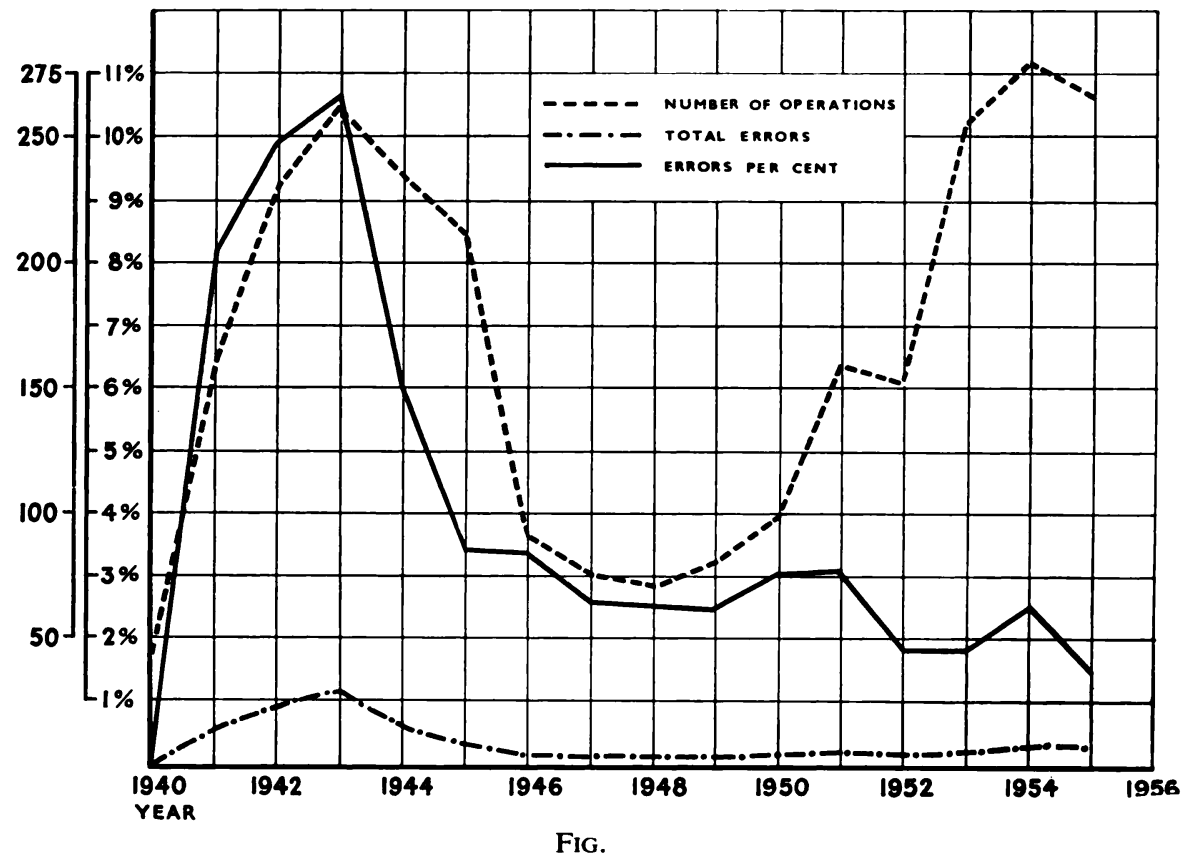

Graph showing total number of operations for suspected cartilage lesions from 1940-1955 inclusive, and the incidence of erroneous diagnosis. The percentage error is also shown.

Errors of localisation (twenty cases)-There were twenty known errors of localisation. In two cases both cartilages were thought to be torn, and in a further thirteen difficulty in localisation was encountered before operation. Of the remaining five cases, one had a discoid lateral cartilage and one was found, after excision of the medial cartilage, to have a torn lateral cartilage with a large tag capable of producing locking signs and symptoms on the medial side. Every error of localisation, with one exception, involved the medial cartilage. The sole error with regard to the lateral cartilage was associated with fibrous union of a fractured tibial spine. In half of the cases either a torn (three) or an attenuated (seven) anterior cruciate ligament was found. In five of these cases damage to the cruciate ligament was recognised before operation.

Thirteen of these errors occurred in the six years 1940-45. In the ten years that followed only seven occurred. This reduction was effected by the establishment of a definite approach to the difficult case which is assessed by a close re-examination of the history in the light of the tendency to make errors on the medial side of the joint, and a planned procedure at 
operation. The examination of the patient may, of course, often solve the difficulty, particularly if the methods advocated by Sir Robert Jones (1924) and by Fisher (1924) are adopted: extension of the knee is necessary to produce localised pain (Jones) and tenderness (Fisher).

If, despite a clear-cut history, no obvious lesion of the cartilage is revealed through an exploratory incision and the cartilage cannot be drawn forwards with a hook, a separate incision is made on the other side. If again there is no obvious lesion, the first cartilage exposed should be excised. This is necessary because the damage may be confined to the

TABLE I

Causes of Wrong Diagnosis in 119 Cases of Arthrotomy for Suspected Cartilage Tears

\begin{tabular}{|c|c|c|c|}
\hline Unreliable witness. & . & . & 32 \\
\hline Error of localisation & . & . & 20 \\
\hline Torn anterior cruciate ligame & & • & 9 \\
\hline Quadriceps insufficiency & . & . & 8 \\
\hline Recurrent subluxation of pat & ella & . & 8 \\
\hline Loose bodies . & . & . & 5 \\
\hline Anterior haemorrhage . & . & . & 4 \\
\hline Chondromalacia patellae & . & . & 4 \\
\hline Abnormalities of fat pad & . & . & 3 \\
\hline Strain of medial ligament & . & . & 2 \\
\hline Slipping semitentinosus tenda & & . & 1 \\
\hline Villous arthritis & . & . & 1 \\
\hline Syphilitic arthritis . & . & . & 1 \\
\hline Sub-sartorial bursa & . & . & 1 \\
\hline Patello-femoral arthritis. & . & . & 1 \\
\hline Old fracture of tibial condyle & & . & 1 \\
\hline Osteochondritis dissecans & . & . & 1 \\
\hline Unexplained. & . & . & 17 \\
\hline
\end{tabular}

periphery of the posterior horn. Every effort should be made to avoid excising both cartilages unnecessarily, because recovery of function may be slow and incomplete.

The occurrence of damage to the anterior cruciate ligament in half of the errors of localisation calls for note. In all these cases there was a history of an acute injury with much swelling, ill-localised pain and tenderness. Instability appearing later may be explained by the cruciate lesion alone or by later injury to the posterior part of the semilunar cartilage. In this way confusion may arise, especially as momentary locking in the presence of a torn anterior cruciate ligament can happen with a smaller cartilage lesion than is possible otherwise.

Routine air arthrography carried out with meticulous attention to detail, and the interpretation of his own radiographs by a surgeon experienced in the technique, may give a high percentage of accuracy (Somerville 1946). Most surgeons will nevertheless consider that the time involved is not justified. A minimal lesion cannot be demonstrated, and unless in use as a routine procedure arthrography would be valueless in the occasional case of difficulty in localisation. This is the opinion of the author and his colleagues. 
Torn anterior cruciate ligament (nine cases)-In nine patients from whom a medial semilunar cartilage was excised in error, isolated lesions of the anterior cruciate ligament were found. In six cases the history was short, varying from one week to one month; the knees were all thought to be locked, with no previous history of instability or locking, and recent tears of the ligament were demonstrated. In two of these examples laxity of the ligament was thought to exist, and examination proved difficult in the other four. This error has occurred only twice since 1945.

In one patient with a history of locking, instability and clicking, the anterior cruciate ligament, in the form of an elongated tag, was hooked forwards from the posterior part of the intercondylar area after excision of a normal cartilage.

In the remaining two patients who presented with instability and medial pain, old-standing damage to the anterior cruciate ligament was found.

Quadriceps insufficiency (eight cases)-Gross wasting of the quadriceps is thought to have been the sole cause of complaints of giving way and pain in eight cases in which a cartilage has been excised in error.

In one patient the obvious effects of old anterior poliomyelitis were to be seen on the other leg, indicating the probability of the cause of the patient's symptoms.

In the other cases incomplete recovery from an injury or operation was thought to be the reason for the quadriceps wasting, and, in turn, of the pain and instability. In three cases previous cartilage operations had been performed three, four and five months before our own exploration. A gunshot wound of the thigh had occurred two months before in two cases, an undisplaced fracture of the tibial condyle eight months before in one case, and an injury two years before, followed by a prolonged stay in hospital, in yet another.

Recurrent subluxation of patella (eight cases)-Seven medial cartilages and one lateral were removed in error when recurrent subluxation of the patella was responsible for the symptoms. On two occasions marked lateral mobility of the patella was noted at operation, and in all of the other cases transplantation of the tibial tubercle was carried out later. Four of these eight errors occurred in females, and these four cases represent half of all the cartilages removed in error from females.

Loose bodies (six cases)-On six occasions no cartilage lesion was demonstrated, but further examination revealed one or more cartilaginous loose bodies. These bodies had not been revealed by previous radiographic examination. In only one patient was the source, in the form of an area of degenerated articular cartilage, discovered at operation. Only one patient gave a history of locking, but all gave a history of injury.

In the absence of positive radiographic findings there was only one example of a frank osteochondritis dissecans lesion as the sole defect.

Anterior haemorrhage (four cases) - In four instances patients were seen with a history of recent injury who, on examination, were considered to have a locked knee but who, at operation, were found to have no lesion of the cartilage but exhibited a large blood clot in the anterior compartment of the joint.

Chondromalacia patellae (four cases)-In two of the four examples of this error there was a clear history of a direct blow, and the records show that tenderness was elicited in the region of the patella. The diagnosis might reasonably have been suspected because there was insufficient evidence to justify a diagnosis of a torn semilunar cartilage.

Abnormalities of the infrapatellar fat pad (three cases)-Fisher (1924) described the anatomy, pathology and symptomatology of anomalies of this structure in some detail, and for some time diagnoses such as "nipping of synovial fringes" and "hypertrophy of the fat pad" have served to disguise a failure to appreciate an underlying cartilage lesion (Smillie 1946). Nevertheless in three cases of the present series the reverse error did occur. On one occasion an enlarged fat pad was thought to be a cystic lateral cartilage, and in another, evidence of a large haemorrhage of some standing was discovered in the fat pad. The third 
case presented a typical clinical picture of a discoid lateral cartilage which proved normal on excision. Closer scrutiny revealed that a large part of the infrapatellar fat pad was being trapped between the anterior cruciate ligament and the lateral femoral condyle.

Syphilitic arthritis (one case)-A young soldier, who injured his knee in a fall from a bicycle three weeks before, presented himself with a swollen painful joint, which on examination was thought to be locked. Biopsy unmistakably showed the syphilitic pathology, although macroscopic appearances were not grossly abnormal.

Villous arthritis (one case) - In this example there was a history of injury and a series of recurrent effusions. The joint was considered to be locked, but operation and biopsy demonstrated the true pathology.

Sub-sartorial bursa (one case)-One medial cartilage was removed in the belief that cystic degeneration of the cartilage was present.

Strain of medial ligament (two cases)-In both cases the history was short, and before operation a cartilage lesion was held in considerable doubt.

Patello-femoral arthritis (one case)-The confusion leading to this error arose from the patient's insistence, despite close questioning, that the joint had locked.

Slipping semitendinosus tendon (one case)-The error was caused by an extra-articular exostosis over which the semitendinosus tendon slipped with a loud snap.

Unexplained errors (seventeen cases)-It is thought that at least four cases, all in females, were in fact examples of recurrent subluxation of the patella. No doubt further errors of localisation are still undiscovered, especially if the other side of the joint was not explored in cases in which there was no apparent difficulty in localisation before operation. It may also be that further malingerers have escaped detection.

It must be stated that the initial inexperience of some of the surgeons has played a part in erroneous diagnosis. Malingering is not always easily uncovered, but the experienced will usually recognise discrepancies in the history and histrionics during examination. The knowledgeable surgeon will accept a history of locking of the knee without previous injury in a miner, but refuse to operate if a cartilage click is the only finding in the footballer whose history contains no evidence of instability or locking. Certainly some of the unexplained errors in this series seem to be due to operation in the face of insufficient evidence.

\section{CONCLUSIONS}

1. It might be considered that error in $\mathbf{4 . 5}$ per cent of meniscectomies is reasonable, but this analysis clearly indicates that many of these mistakes are avoidable. The reduction in error over the past ten years supports this statement.

2. The highest incidence was due to malingering, but this problem is not of great moment under peace-time conditions, which afford opportunity for delay in diagnosis and the use of rehabilitation units.

3. There is an obvious need for careful assessment in cases in which there is doubt about the localisation of the lesion. The marked prevalence of errors on the medial side of the joint, and a planned approach to the operation are of great importance.

4. On fourteen occasions errors were made in cases with a history of recent primary injury, and in each instance the joint was thought to be locked. The emphasis in the differential diagnosis would appear to lean heavily towards lesions of the anterior cruciate ligament and haemorrhage in the anterior part of the joint. This serves to emphasise that great care is required in the assessment of recent injuries. A more cautious attitude is necessary, and, when the integrity of the anterior cruciate ligament is in doubt, examination under anaesthesia is always justifiable and sometimes necessary.

5. Recurrent subluxation of the patella is a source of error that should be noted, especially in women. Smillie (1950) stated that "errors of diagnosis occur most frequently in the female. It is hardly an exaggeration of the situation to suggest that every internal derangement 
of the knee joint in a woman should be suspected to be a recurrent subluxation of the patella until proved otherwise; tears of the meniscus are rare."

6. Caution must be the keynote when gross quadriceps wasting is the main feature. The surgeon must ensure that as good a recovery as possible has been made after previous injury before further operation is contemplated.

\section{SUMMARY}

1. In the course of 2,668 operations for suspected cartilage lesions 119 errors of diagnosis were revealed.

2. These errors are analysed and certain conclusions are drawn.

My thanks are due to my senior colleague, Mr I. S. Smillie, Director of Orthopaedics for the Eastern Region of Scotland. Much of the material used in this investigation has already been the subject of his widespread researches into the traumatic pathology of the knee joint. Mr Smillie has always been and remains a most stimulating mentor, and I cannot overstress my gratitude to him. I would like also to thank other colleagues, past and present, for the use of their clinical material.

\section{REFERENCES}

Annandale, T. (1885): An Operation for Displaced Semilunar Cartilage. British Medical Journal, i, 779.

BRISTOw, W. R. (1935): Internal Derangement of the Knee Joint. Journal of Bone and Joint Surgery, 17, 605. DunN, N. (1934): Observations on Some Injuries of the Knee-joint. Lancet. i, 1,267.

Fisher, A. G. T. (1924): Internal Derangements of the Knee-joint, pp. 16, 41 and 127. London: H. K. Lewis \& Co. Ltd.

HeY, W. (1803): Practical Observations in Surgery, p. 327. London: T. Cadell and W. Davies.

Hippocrates (1849): The Genuine Works. Translated by F. Adams. Volume 2. London: The Sydenham Society.

JONES, Sir R. (1924): Note 5 in A. G. T. Fisher's Internal Derangements of the Knee-joint, p. 189.

MARTIN, A. M. (1913): Injuries to Semilunar Cartilages. Proceedings of the Royal Society of Medicine, Surgical Section, 6, 23.

Paré, A. (1841): Oeuvres Complètes. Tome 3, p. 32. Paris: J. B. Baillière.

Smillie, I. S. (1946): Injuries of the Knee Joint, p. 287. Edinburgh: E. \& S. Livingstone Ltd.

Smillie, I. S. (1950): Injuries of the Knee Joint. Second edition, pp. 101, 250. Edinburgh: E. \& S. Livingstone Ltd.

Somerville, E. W. (1946): Air Arthrography as an Aid to Diagnosis of Lesions of the Menisci of the Knee Joint. Journal of Bone and Joint Surgery, 28, 451. 